Estudios sobre armas antiguas, arte militar y vida cultural en oriente y occidente XXXIII (2013), pp. 113-130

ISSN : 0436-029X

doi: $10.3989 /$ gladius.2013.0005

\title{
TÉCNICAS DE ATAQUE Y DEFENSA EN LOS ASEDIOS DEL SIGLO XIII: ÁMBITO CATALANO-ARAGONÉS Y OCCITANO
}

\author{
OFFENSIVE AND DEFENSIVE TACTICS IN 13TH CENTURY SIEGES: THE CASES OF \\ ARAGÓN-CATALONIA AND OCCITANIA
}

POR

JoSEP SuÑÉ ARCE*

\section{RESUMEN - ABSTRACT}

En las últimas décadas, el estudio sobre los asedios medievales ha conocido una expansión importante, especialmente entre los historiadores franceses y anglo-sajones, a través de los cuales se conocen abundantes datos sobre las guerras transcurridas en ambas áreas culturales para un periodo cronológico extenso. Sin embargo, no se puede decir lo mismo ni respecto al espacio catalán-aragonés y occitano ni para el siglo trece en concreto. Este artículo ofrece algunas de las técnicas de ataque y defensa para los asedios de este mismo periodo y área geográfica, a través del análisis de quince asedios que aparecen en las fuentes catalanas y occitanas. El estudio muestra que en el siglo trece había una gran variedad de técnicas para expugnar ciudades, y la relevancia de ballesteros e ingenieros en la guerra medieval.

In the last decades, the study of medieval sieges has met an important expansion, especially among French and Anglo-Saxon historians, whereby are know very details about wars elapsed in both cultural areas for an extensive chronological period. However, can not say the same neither respect to Catalan-Aragonese and Occitan space nor for the thirteen century specifically. This article offers some attacks and defense techniques for the sieges of this same time period and geographical area, through an analysis of fifteen sieges appeared in Catalan and Occitan sources. The study shows that in thirteen century there was a great variety of techniques to take by storm cities, and the relevance of crossbowmen and engineers in medieval war.

\section{Palabras Clave - Keywords}

Siglo trece; asedios; trabuco; Gatas; Castillos de madera; Minas; Picos; Asalto con escaleras; Líquidos inflamables; Hostigamiento; Defensa de ciudades; Ballesteros; Ingenieros; Catalanes; Aragoneses; Occitanos.

Thirteen century; Sieges; Trebuchet; Cats; Belfries; Mining; Picks; Assault with ladders; Flammable liquids; Harassment; Defense of cities; Crossbowmen; Engineers; Catalans; Aragoneses; Occitans.

\section{INTRODUCCIÓN}

Aquello que caracteriza y diferencia la guerra medieval de la de cualquier otro período histórico es la proliferación e importancia que en ésta tuvieron los asedios, tanto a ciudades como a castillos. A lo largo de la Edad Media, estas acciones militares sobrepasaron en número a las

* Departamento de Historia Medieval, Paleografía y Diplomática. Facultad de Filosofía, Geografía e Historia. Universidad de Barcelona, cives809@gmail.com. 
de cualquier otro tipo, ya fuesen batallas campales, enfrentamientos navales o expediciones de ataque a caballo (Jones, 2010: 212). Esta última afirmación puede parecer exagerada, aunque cobra sentido si se incluyen las campañas de saqueo y destrucción entre las operaciones preparatorias de un asedio (García Fitz, 2001: 171-172). Los estudios sobre asedios medievales han proliferado a lo largo del pasado siglo y llegan hasta la actualidad (Bradbury, 1992; Contamine, 1984; Corfis y Wolfe, 1995; Higham y Barker, 1992; Purton, 2009, 2010; Rogers, 1992). Autores anglosajones y franceses han analizado sus más diversos aspectos a lo largo de un vasto período cronológico que va desde la conquista de Barcelona a manos de las tropas carolingias (801) hasta el espectacular asedio de Constantinopla (1453), descrito de manera pormenorizada y brillante por Sir Steven Runciman en un importante trabajo (1965). Gracias a estos autores no faltan datos sobre los asedios practicados durante la historia medieval de los territorios anglosajones y franceses. Así pues, las acciones bélicas transcurridas en el contexto de la conquista normanda de Inglaterra o del eterno conflicto entre Plantagenets y Capetos, sólo por poner dos de los ejemplos más representativos, han recibido merecidamente una atención privilegiada (Hill y Rumble, 1996; Morillo, 1994; Prestwich, 1996). Lo mismo se puede decir para el espacio hispano y, más concretamente, castellano-leonés, donde en los últimos años han aparecido con fuerza estudios sobre esta temática (Bruhn de Hoffemeyer, 1982; Navareño Mateos, 1998:575-592; García Fitz, 1997:38-41, 2001, 2000:115-154, 2005:219-254; Ladero Quesada, 2010). Sin embargo, no se puede afirmar lo mismo en relación al ámbito catalano-aragonés y occitano, puesto que, si bien los asedios sucedidos durante la Cruzada Albigense (1209-1218) han estado tratados en estas mismas obras o en otras más específicas (Compan, 1989:45-61; Hélas, 2001:51-60; Marvin, 2001:373-395, 2008; Zambon, 2006:2437; Alvira Cabrer, 2002, 2010:59-72), poca atención han recibido en cambio aquéllos que se realizaron en el contexto de las guerras de expansión impulsadas por los reyes de Aragón del linaje de Barcelona a través del levante peninsular y Mediterráneo durante el siglo XIII. Por lo general, estos dos conflictos se han tratado por separado, obviando que los que empuñaban las armas eran en muchos casos los mismos actores pero bajo diferentes banderas, liderazgos y contextos (Alvira Cabrer, 2002:537, 545, 570). Asimismo, tampoco abundan trabajos que centren su análisis en un período cronológico determinado, bien al contrario lo que ha prevalecido son estudios de conjunto que profundizan poco en las características de una etapa concreta, impidiendo observar a largo plazo la evolución de las tácticas, armamento y técnicas utilizadas en los asedios medievales de la manera más clara y completa posible. Ciertamente, autores como L. Monreal y Tejada (1971), P. D. Humphries (1985:173-178) y P. E. Chevedden (1996:47-94; 2011:313-339) han analizado algunos de los aspectos más destacados de la poliorcética catalana y aragonesa, pero sus estudios, o van más allá del siglo XIII, o están centrados únicamente en algunas de las armas más utilizadas en estos asedios, como por ejemplo la artillería de tracción y contrapeso.

Estas dos razones justifican la realización del presente trabajo, que pretende estudiar las técnicas de ataque y defensa utilizadas en los asedios del siglo XIII en el ámbito occitano, catalán y aragonés. Para lograr tal propósito se han estudiado los asedios de Béziers (1209), Termes (1210), Lavaur (1211), Moissac (1212), Le Pujol (1213), Muret (1213), Beaucaire (1216) y Tolosa (1211/1217-1218), producidos en el contexto de la Cruzada Albigense, los de Mallorca (1229), Borriana (1232) y Valencia (1238), realizados por Jaime I de Aragón (12081276) durante la conquista de los reinos musulmanes de Mayurqa y Balansiya, los de Montesquiu d'Albera (1285), Llers (1285) y Girona (1285), llevados a cabo por Felipe III el Atrevido de Francia (1245-1285) en su desastrosa cruzada contra Pedro III el Grande de Aragón (12401285) y, finalmente, el de Gallipoli (1306), acontecido durante la estancia de la Compañía Catalana de Oriente en tierras bizantinas a inicios del siglo XIV. Los datos de estos asedios apa- 
recen documentados en cuatro importantes obras medievales, la Canso de la Crozada, escrita por Guillermo de Tudela y un autor anónimo posterior a éste, el Llibre dels Feits del rey Jaime I de Aragón, la Crònica o Llibre del rei En Pere de Bernat Desclot y la Crònica de Ramón Muntaner. Si para la primera de éstas, vital para el conocimiento de la Cruzada Albigense, se ha trabajado con la edición de E. Martin-Chabot y la traducción que recientemente han publicado V. Martines y G. Ensenyat, para las otras tres, que dan una información de primera mano para la conquista de Mallorca y Valencia, la cruzada de Felipe III de Francia contra Cataluña y la campaña de los almogávares a Oriente, se han consultado las ediciones de F. Soldevila, revisadas apenas hace unos años por J. Bruguera y M. T. Ferrer Mallol, y la nueva edición de S. M. Cingolani. Las dificultades para resolver el significado de algunos términos específicos aparecidos en estas fuentes se han salvado recurriendo al Diccionari català-valencià-balear del Institut d'Estudis Catalans. Asimismo, se ha cuidado de traducir al castellano cada una de las citas publicadas en este artículo. La información procedente del vaciado de fuentes se ha agrupado en apartados que pretenden reconstruir, paso a paso, lo que podría haber sido un asedio modelo, dejando constancia que en la realidad no tenían porqué producirse cada una de las fases aparecidas en este trabajo. De igual modo, el lector comprobará que el trabajo se ha dividido en dos partes, una primera dedicada a las acciones ofensivas, y una segunda que analiza las defensivas.

\section{EL ATAQUE A UN CASTILLO O A UNA CIUDAD FORTIFICADA}

Cuando un ejército de la Plena Edad Media formado por unos centenares o pocos miles de combatientes se encontraba con una oposición en forma de punto fortificado, torre, castillo, villa o ciudad, tenía varias maneras de superarla. Todo dependía del tiempo que tuviese en relación a los suministros disponibles, de la accesibilidad al lugar en cuestión o de su tamaño, puesto que no era lo mismo atacar una torre situada en un lugar llano que una situada encima de un monte escarpado, o expugnar una ciudad de miles de habitantes en vez de una simple casa fuerte. También se debía tener en cuenta el potencial de la guarnición que había en el lugar asediado. Ahora bien, aparte de estas consideraciones previas, normalmente los ejércitos atacantes actuaban de una forma muy parecida a la vista de las fuentes consultadas.

\subsection{Primeras ACCIONES DE APROXIMACIÓN: EL TANTEO}

En primer lugar, parece que era normal realizar un primer intento de asalto, no en toda regla, sino más bien como una forma de medir la fuerza del contrincante. Si la resistencia de los asediados era muy débil podía derivar en un ataque definitivo, pero a la vista de las fuentes consultadas se debe concluir que esto sucedía sólo cuando en el lugar atacado había carencias defensivas, o bien cuando la guarnición era escasa. Incluso en ocasiones da la sensación que este ataque de tanteo se hacía en paralelo a la construcción del campamento. Este ataque lo llevaban a cabo caballeros bien armados que, acercándose a las murallas de la ciudad o castillo, buscaban las puertas para intentar apoderarse de una de ellas y poder penetrar en el recinto amurallado, siempre y cuando desde dentro no saliese ningún contingente para abortar la operación. Así lo explica el autor anónimo de la Canso de la Crozada (14:139) cuando relata la batalla de Muret (1213), quien afirma que los caballeros catalanes, aragoneses y occitanos lanzaron sus lanzas contra los cruzados que les impedían el acceso por una de las puertas que todavía permanecía abierta, produciéndose una intensa lucha. Algunos jinetes parece que incluso llegaron a entrar en la villa, aunque la acción no tuvo ninguna trascendencia para el desarrollo posterior (Alvira Cabrer, 2002:291). En el asedio de Girona del año 1285, pocos 
días después de levantar el campamento, y tras un intento fallido de negociar con el capitán de la guarnición, los cruzados franceses enviaron 400 caballeros al ataque en dirección a la puerta de la judería, siendo interceptados por contingentes de la guarnición y obligados a retirarse con fuertes pérdidas después de un duro combate, según el testimonio de Bernat Desclot, autor de la Crònica o Llibre del rei En Pere (cap.156). Normalmente, si este primer ataque fracasaba se procedía al asedio en toda regla, aunque había ocasiones en las que, a pesar de un primer asalto rechazado, los atacantes no se amedrentaban y seguían con la misma táctica. Casos como éste se encuentran documentados en esta misma obra cuando se relatan las acciones realizadas por los cruzados franceses en el Rosselló y Empordà. Si bien al castillo de Montesquiu se le dieron hasta cuatro batallas en un solo día antes de desistir en el intento, para conseguir la capitulación del de Llers, relata Bernat Desclot que se realizaron hasta catorce asaltos con caballeros y hombres a pie (caps. 140 y 154).

\subsection{LA INSTALACIÓN DEL CAMPAMENTO}

Una vez iniciado el asedio en toda regla era de vital importancia la ubicación del campamento. Se buscaba que no estuviese expuesto a las ballestas o a la artillería de la plaza atacada, como también era deseable que se instalase en un lugar elevado o de fácil defensa por si los asediados lanzaban un ataque sorpresa. Tal como se recoge en el Llibre dels Feits (cap. 69), si esto no era posible se le protegía con un foso y una empalizada de madera. Otra consideración a tener en cuenta era garantizar un fácil avituallamiento de la hueste asediadora, tanto por lo que respecta a los alimentos como al agua dulce. En esta misma obra (cap. 67) se explica que Jaime I durante el asedio de Mallorca (1229) estableció su campamento cerca de la playa para facilitar el avituallamiento desde los barcos y que una acequia lo partía en dos mitades, gracias a la cual se puede deducir que las tropas catalanas y aragonesas no carecieron de agua dulce durante el sitio.

\subsection{LA INSTALACIÓN DE LA ARTILLERÍA}

Una vez instalado el campamento se procedía al montaje de las armas de asedio propiamente dichas. Si alguna de ésas aparece reiteradamente documentada en las fuentes catalanas y occitanas del siglo XIII, ésta es el trabuco o trabuquete, más conocido con el nombre de trebuchet. En el cuaderno de Villard de Honnecourt (1220-1240) hay un dibujo de esta máquina acompañado de la información siguiente (Lám.59:137): «Observad los pies [soles] sobre los que se asienta en tierra. Ved delante los dos tornos de eje vertical [windas] y la cuerda desplegada con la que se tensa la vara. Podéis verlo en esta otra página. Hay que izar un gran peso, pues el contrapeso es muy pesado, y consiste en un canasto lleno de tierra» ${ }^{1}$. En concreto, el trabuquete descrito en estas líneas era uno de contrapeso, que correspondía a la artillería más pesada y potente de su tiempo. Los pies de los que habla el texto eran dos armazones en forma triangular, encima de los cuales se montaba un eje por el que debía pivotar una larga viga o vara (Chevedden, 2011: 314-315). A causa del eje, ésta se veía dividida en un brazo corto y otro largo. En el primero se encontraba el contrapeso y en el segundo una honda que tenía dos cuerdas. Una de ellas estaba firmemente atada al final de la viga, mientras que la otra colgaba de una estaca de acero que sobresalía también de la vara. Una vez introducido el proyectil en

1 He modificado el texto de la traducción indicándolo en cursiva. En vez de anclajes escribo pies y en vez de resortes escribo tornos de eje vertical, términos que me parecen más ajustados al sentido real del texto. Véase: R. Martin (2012). 
la honda se dejaba caer el contrapeso. Cuando la viga alcanzaba el ángulo apropiado, la cuerda de la honda que colgaba de la estaca se liberaba del lazo y arrojaba el proyectil. Experimentos modernos han demostrado la enorme capacidad destructiva de este aparato. En Inglaterra, un trabuquete lanzó $476 \mathrm{~kg}$ de peso a 80 metros de distancia con un contrapeso de 30 toneladas (Chevedden et alii, 1995:66-71). Tal como se afirma en el texto, para tensar la vara con la cuerda desplegada se debía levantar un peso enorme, por lo que se realizaba con la ayuda de dos tornos de eje vertical (García Fitz, 2011:823). Sin embargo, el trabuquete de contrapeso no era el único que existía en el siglo XIII, sino que convivía con el de tracción y el híbrido (Chevedden, 2011: 320-321). Este primero sustituía el contrapeso por unas cuerdas de las que tiraban un equipo de hombres, mientras que de la honda se colgaba uno de ellos que conseguía flexionar la viga con su cuerpo, aumentando así el alcance del proyectil cuando era disparado (Chevedden et alii, 1995: 66-71). El segundo era una mezcla de tracción y contrapeso, recibiendo el equipo de hombres que tiraba de las cuerdas una ayuda gravitatoria. Aunque carecían de la potencia devastadora del trabuquete de contrapeso, tenían la ventaja de poder ser disparados de forma más rápida (Chevedden, 2011: 325). Datos sobre este tipo de artillería se encuentran documentados en la Canso de la Crozada (35: 204), donde se afirma que después de introducir una piedra en la honda se estiraba de las cuerdas para provocar el lanzamiento ${ }^{2}$.

En el Llibre dels Feits, según P. E. Chevedden (1996: 68-76; 2011: 319-329), la algarrada haría referencia a un trabuquete de tracción con pie en forma de poste, mientras que el almajanech (almajaneque) y el manganel corresponderían también a trabucos de tracción pero con pies en forma de armazón triangular. El fenèvol (fundíbulo), en cambio, sería la versión híbrida a la que se ha hecho referencia anteriormente ${ }^{3}$. El trabuquet correspondería a un trabuquete de contrapeso con pies en forma de armazón triangular, mientras que la brigola sería también un trabuco de contrapeso pero con un pie en forma de poste y dos cajas de contrapeso en vez de una.

Toda esta maquinaria no se fabricaría en el lugar del asedio sino que se trasladaría de un sitio a otro dividida en partes para facilitar su transporte. Al menos esto es lo que se puede deducir del relato que hace la Canso de la Crozada (5: 53) del asedio que protagonizó Simón de Montfort contra el castillo de Termes en el año $1210^{4}$. La finalidad principal de esta maquinaria era bombardear los muros y las torres de la ciudad o castillo asediado para debilitar su estructura, tal como aparece bien recogido en el Llibre dels Feits (cap. 175): «...que subiesen por aquella torre que había derribado el fundíbulo». El bombardeo se hacía de forma continuada e intensa. En este sentido, las crónicas consultadas sólo indican cuando utilizan por primera vez un trabuquete para a continuación enmudecer al respecto, como si se diese por hecho que el bombardeo a partir de aquel instante sería un acto cotidiano e innecesario de ser recordado por escrito. Esto quiere decir que, más allá de destruir los muros, los trabuquetes y otras maquinas de tiro tenían una función psicológica, muy explícita en ocasiones según el Llibre dels Feits (cap. 70), donde se puede leer que el cráneo de un cabecilla musulmán fue lanzado dentro de las murallas de la ciudad de Mallorca (Monreal y Tejada, 1971: 35). Los trabuquetes también servían para introducir dentro de las ciudades cuerpos muertos con la intención de propagar enfermedades entre los defensores (Contamine, 1984: 130). Las fuentes reflejan que eran máquinas muy apreciadas y complejas, sólo capaces de ser construidas por unos cuantos maes-

2 Aparte de los trabajos de P. E. Chevedden sobre artillería medieval, también se pueden consultar los de J. F. Finó (1972: 25-43), D. R. Hill (1973: 99-115), C. M. Gillmor (1981: 1-8), D. J. C. King (1982: 457-469) y R. Rogers (1992: 266-273).

3 Este instrumento podía realizar un promedio ligeramente superior al de un tiro por minuto según L. Monreal y Tejada (1971: 23).

4 Para el asedio de Termes (1210) véase: G. Langlois (1994: 101-134; 1995: 87-89; 2005: 53-60) y L. W. Marvin (2008: 84-93). 
tros, algunos de los cuales han conservado su nombre para la posteridad, como por ejemplo el maestro Nicoloso, recogido en el Llibre dels Feits (cap. 157).

Entre el campamento y el lugar exacto donde se instalaban los trabuquetes podía haber una distancia considerable. Esto era debido a que el campamento se construía fuera del alcance de los ballesteros que defendían la ciudad o castillo. A lo largo de las fuentes consultadas se diferencia el lugar donde están las tiendas del sitio ocupado por los instrumentos de guerra. Para que fuese efectivo realmente un trabuquete no se debía montar demasiado lejos del objetivo que se quería atacar, por lo que quedaba al alcance no sólo de los trabuquetes que defendiesen la ciudad o castillo, sino también de los ballesteros. Este hecho obligaba a proteger los trabuquetes de las flechas de los defensores, tal como se explica de una forma un tanto exagerada en el Llibre dels Feits (cap.159): «...tanto habíamos cubierto de cledes el fundíbulo que era bajo... ». Las cledes deben ser interpretadas como unas estructuras defensivas móviles construidas con ramas y postes de madera, gracias a las cuales los atacantes se podían proteger de las flechas, dardos y piedras de los defensores (Monreal y Tejada, 1971: 14). De la misma manera, puesto que el campamento se encontraba relativamente alejado de las máquinas de asedio, se hacía necesaria la presencia permanente de combatientes para que las vigilasen y protegiesen de los posibles contragolpes de los defensores, tal como se describe en el Llibre dels Feits (cap. 82).

\subsection{El BLOQUeo De LA PLAZA aSEDiada}

En paralelo a la instalación de la artillería se procedía al bloqueo de la ciudad o castillo asediado. El objetivo era impedir la llegada de cualquier tipo de socorro a la plaza sitiada, ya fuese una columna de avituallamiento para evitar el hambre entre los defensores o tropas de refuerzo para aumentar la capacidad defensiva de la guarnición. De hecho, muchas de las batallas medievales se producían cuando el ejército sitiador intentaba interceptar la llegada de refuerzos a la plaza asediada, ejemplos de esto se encuentran documentados tanto en la Canso de la Crozada (14: 137-140) como en la Crònica de Bernat Desclot (cap. 2), para referirse respectivamente a las batallas de Muret (1213) y Fraga (1134). Con frecuencia, a fin de garantizar que no entrasen avituallamientos para los defensores y para que los atacantes pudieran abastecerse, se procedía a un saqueo intensivo del área cercana a la fortaleza o ciudad, tal como aparece narrado en la Canso de la Crozada (29: 189). Si se tiene en cuenta que buena parte de las ciudades capitulaban por la ausencia de recursos, se debe entender que ésta era quizás una de las operaciones más importantes de un asedio (García Fitz, 2001: 241).

\subsection{LAS OPERACIONES PARA EXPUGNAR UNA PLAZA}

En el caso de no haber logrado la capitulación inmediata de la plaza asediada, se procedía a su expugnación de manera violenta. Puesto que el objetivo era superar el foso y la muralla que rodeaban la villa o castillo, existían dos maneras de lograrlo, o bien se burlaba el sistema defensivo por arriba mediante un asalto con escaleras que permitiese el acceso al camino de ronda para apoderarse de alguna de las puertas, o se conseguía su destrucción a través del derribo de la muralla. Del primero de los métodos descritos se han encontrado pocas referencias documentales, quizá porque las pérdidas en hombres en un asalto de estas características debían ser elevadas como también escasas sus posibilidades de éxito. Según la Crònica o Llibre del rei En Pere escrito por Bernat Desclot (cap.164), durante el asedio de Girona del año 1285 los cruzados franceses intentaron expugnar esta ciudad de ese modo consiguiendo un sonado 
fracaso. El procedimiento era muy sencillo, las escaleras de madera se apoyaban en la muralla que se quería asaltar y de esa manera se superaba el vacío del foso. Sin embargo, mucho más frecuente era el otro sistema de ataque, el de derribar la muralla.

\subsubsection{El derribo de la muralla}

\subsubsection{Picar los muros}

Había diversas maneras de derribar una muralla. Una de las más habituales consistía en lanzar una gran cantidad de hombres armados con mazos, martillos y picos dentro del foso para que golpeasen los paramentos del muro hasta migar una o varias piedras y provocar en consecuencia la caída de la muralla. Así aparece relatado en la Canso de la Crozada para el asedio de Béziers del año 12095 (2: 19): «En camisas y bragas comienzan a ir / al entorno de toda la villa, para los muros derribar; / dentro de los fosos entran y empiezan a picar, / y los otros las puertas romper y trocear». Sin embargo, esta acción sólo se podía conseguir si el foso era poco profundo. Al no ser así era necesario aplanarlo mediante el uso de materiales naturales como ramas y tierra que recibían el nombre de pertrechos (pertrets), gracias a los cuales los hombres podían picar a una altura razonable de la muralla. Jaime I describe en el Llibre dels Feits (cap. 73) que, para conquistar la ciudad de Mallorca, aplanó el foso alternando capas de leña y tierra. En la Canso de la Crozada (13: 134) se dice que en el asedio de Le Pujol del año 1213 los caballeros, burgueses y sirvents occitanos, catalanes y aragoneses llevaban un fajo de leña sobre los hombros para, una vez cerca del foso, lanzarlo abajo y facilitar el posterior trabajo de picar la muralla ${ }^{6}$. Tal como aparece expresado en las fuentes da la sensación que desde arriba de las murallas se podía hacer poca cosa para contrarrestar la acción de los picadores. En esto tendría mucho que ver la acción de los ballesteros, que dispararían contra los defensores que asomaban sus cuerpos para lanzar objetos contundentes o líquidos inflamables abajo, aunque también se buscaba intencionadamente aquellos puntos muertos de la muralla que quedaban fuera del alcance de la guarnición, tal como se recoge en el Llibre dels Feits (cap. 262) cuando se describe el asedio a la ciudad de Valencia (1238) ${ }^{7}$.

\subsubsection{Minar la muralla}

Otro sistema para derribar una muralla era el de minar una torre. En esencia consistía en excavar un agujero en el suelo sin ser vistos por los defensores, para desde allí hacer un túnel que llegase hasta los pies de la torre. Una vez debajo de la muralla se excavaban los cimientos del muro. Para evitar un hundimiento precipitado se sostenían los cimientos con puntales. Cuando la obra ya estaba acabada se prendía fuego a los puntales y la torre se venía abajo (Monreal y Tejada, 1971: 25). Así lo explica Jaime I en el Llibre dels Feits (cap.72) cuando relata el asedio de Mallorca del año 1229. Las fuentes en cambio no describen cómo se provocaba el fuego en los puntales, aunque es posible que previamente fuesen untados con brea o alquitrán y puestos en contacto con un importante trozo de estopa, para que encendido el fuego en ésta, tuviesen tiempo de huir los que estaban dentro de la mina. Sin embargo, no siempre

\footnotetext{
5 Para este asedio véanse los trabajos de J. Berlioz (1994), M. Meschini (2005: 25-38), L. W. Marvin (2008: 37-45) y M. Bourin (2010).

6 El asedio de Le Pujol (1213) ha sido estudiado por J. B. Vazeille (1962-1963: 124-132) y L. W. Marvin (2008: 172-174). El término occitano sirvent parece corresponderse con el servent catalán, que hace referencia a un combatiente de a pie. Véase A. M. Alcover y F. de B. Moll (2001-2002).

7 Más información sobre este asedio se puede encontrar en P. E. Chevedden (2011: 335-336).
} 
se tenía que incendiar los puntales para provocar el derrumbe de la torre. Esta misma acción se conseguía derribándolos con la ayuda de una gúmena, tal como se recoge en el Llibre dels Feits (cap. 72). Todavía en este asedio de Mallorca aparece otro tipo diferente de mina que, en vez de ir bajo tierra, lo hacía por encima del suelo (Monreal y Tejada, 1971: 27). En concreto, vuelve a ser el Llibre dels Feits (cap. 69) quien informa que el occitano Jaçbert de Barberà construyó un camino cubierto que permitía a los sitiadores acceder hasta el foso sin peligro de ser heridos por el efecto de dardos o piedras lanzados por los defensores. El elemento clave para su construcción fue un mantelete que iba sobre ruedas y que estaba formado por cledes de tres dobles de buena madera. Por lo que continua diciendo el texto, se intuye que con la protección de esta máquina los combatientes catalanes y aragoneses fueron clavando palos de madera en el suelo, uno al lado del otro, para unir o atar respectivamente sus cabezas y a continuación cubrirlos con ramas y tierra. Este camino cubierto llegaba hasta el foso, pero parece ser que para minar la torre debían salir hombres de él para apuntalar los cimientos o picar los muros tal como se ha visto anteriormente. Hablar del mantelete ayuda a introducir el tema de la protección de los atacantes cuando se aproximaban a la muralla.

\subsubsection{Maquinaria para proteger los asaltantes}

Si bien parece que era relativamente sencillo picar o apuntalar los muros de las ciudades o castillos asediados, no lo era en cambio salvar la distancia que había entre el campamento y el foso. Asimismo, las minas no se podían empezar a excavar demasiado lejos de la muralla para evitar los excesos en esfuerzos de obra o la realización de agujeros a la superficie que podían ser detectados por la guarnición. Por lo tanto, ya fuese para picar o minar se hacía necesario proteger al sitiador. Los casos que han aparecido en el apartado anterior, en los que se documenta la acción de manteletes y cledes, no son ni mucho menos los únicos que ofrecen las fuentes consultadas para este mismo periodo. En el primer asedio de Tolosa del año 1211, los cruzados hicieron servir tarjas de cuero a modo de manteletes o cledes según la Canso de la Crozada (8: 80), gracias a las cuales se pudieron proteger los soldados y lanzar los pertrechos al foso ${ }^{8}$. Pero detrás de un mantelete no sólo se protegían guerreros sino que también se excavaban las minas, tal como se relata en el Llibre dels Feits (cap. 69) durante el asedio de la ciudad de Mallorca (1229). De nuevo en esta obra (caps. 170 y 175) se documenta la misma operación pero para el asedio de Borriana $(1232)^{9}$. Si en un primer momento se dice esto: «... que ordenéis hacer cledes a los concejos hasta trescientas, y yo iré a meterlas, y mi compañía, cerca del foso», después se dice lo siguiente: «...más adelante fueron las minas hechas que salían al foso... ». Sin embargo, cledes y manteletes no eran los únicos instrumentos que permitían a los sitiadores acercarse a la muralla sin recibir daño, había todavía otros más sofisticados y polivalentes como se podrá ver a continuación.

\subsection{Las gatas}

Una gata era una contra fortificación. Consistía en una gran estructura de madera y cuero vaciada por dentro para que los combatientes pudiesen estar seguros en su interior. Si bien en general las fuentes consultadas no precisan su forma, parece que era una estructura muy compleja, como se puede apreciar en un fragmento de la Crònica o Llibre del rei En Pere (cap. 162) escrito por Bernat Desclot, donde se afirma que estaban hechas de madera y recubiertas

8 Para este asedio véanse los trabajos de M. Roquebert (2001: 406-418) y L. W. Marvin (2008: 111-116). Sobre la función de las tarjas véase: (Monreal y Tejada, 1971: 15).

9 Más datos para el sitio de Borriana (1232) en P. E. Chevedden (2011: 334). 
con cuero para protegerlas del fuego. Incluso parece ser que las partes más críticas de la estructura, aquéllas más expuestas a los ataques de los defensores, estaban protegidas por piezas de hierro o acero, tal como se recoge en la Canso de la Crozada (33: 200). Algunas de estas máquinas podían llegar a ser muy grandes. En el mismo capítulo de esta obra, ambientado en el asedio de Tolosa por parte de Simón de Montfort (1218), la gata construida podía acoger hasta 400 caballeros -desmontados- y 150 arqueros $^{10}$. La función de la gata era similar a la que hacían los manteletes y cledes. Cuando Bernat Desclot (cap. 162) relata el asedio de Girona del año 1285, afirma que la función de las gatas era la siguiente: «...de manera que uno se podía acercar al muro de la ciudad para empezar la mina más cerca... ». Lo mismo se puede deducir de la información que da la Canso de la Crozada (6: 68) para el asedio de Lavaur (1211) ${ }^{11}$ : «La gata situaron al fondo del foso, / y lanzaron los pertrechos y tanto cavaron / que los de dentro se rindieron, porque son presos y forzados». Parece que la finalidad de la gata era proteger a los que hacían una mina o a aquéllos que lanzaban pertrechos al foso. Ahora bien, como ya se ha dicho, las gatas eran muy polivalentes y podían cumplir otras funciones como hostigar a los defensores de la muralla. Por ejemplo, en la Canso de la Crozada (34: 202) se afirma que la gata construida en ocasión del asedio de Tolosa (1218) tenía la finalidad de lanzar fuego griego contra los defensores de la ciudad. Quizá por esta polivalencia, da la sensación que las gatas eran unos instrumentos muy temidos por los sitiados.

\subsection{Los arietes}

Otro instrumento utilizado en los asedios del siglo XIII eran los arietes. Sin embargo ni se describe cómo se hacían ni en qué consistían. Lo único que aparece en la Canso de la Crozada (11: 121 y 19: 164) es que se trataba de una estructura que disponía de una viga de madera con la que se golpeaba los muros de las ciudades para provocar su derribo ${ }^{12}$.

\subsection{Los castillos de madera}

Otro instrumento muy utilizado, y del que sí se tiene información precisa, es de los castillos de madera. Jaime I explica en el Llibre dels Feits (cap.158) cómo era el que hizo construir durante el asedio de Borriana (1232): «El castillo de madera tendrá dos marcos en cada parte, y serán cuatro, aparte de dos más que habrá en la frontera de cada parte, delante y detrás, y éstos cerraran los marcos, y les haremos dos suelos, uno en la mitad del castillo, y el otro en lo más alto: y en lo alto la mitad serán ballesteros y [la otra mitad] hombres que apedrearán a aquellos sarracenos que subirán al muro... ». El texto hace referencia a los marcos o paredes y a los suelos. De marcos o paredes había un total de ocho, dos y dos en cada lado -se sobrentiende que uno iba encima del otro para ganar altura-, dos adelante y otros dos detrás, formando un cuadrado o rectángulo. De suelos, en cambio, había sólo dos, uno a la mitad de la estructura y otro haciendo de tejado. Probablemente, los marcos o paredes de madera estarían cubiertos con pieles mojadas, que protegiesen el castillo del fuego lanzado por los sitiados. A pesar del imaginario popular, este castillo no se movía mediante ruedas sino que lo hacía a través de parales, que eran postes de madera colocados en el suelo uno en paralelo al otro para que las

10 El segundo asedio de Tolosa (1217-1218) ha sido estudiado entre otros por M. Roquebert (2001: 1078-1121), C. Peytavie (2005: 105-124) y L. W. Marvin (2008: 268-296).

11 Sobre éste puede consultarse el trabajo de L. W. Marvin (2008: 98-105).

12 En concreto, están documentados en los asedios de Moissac (1212) y Beaucaire (1216). Más información para el primero de éstos en L. W. Marvin (2008: 147-153). Para el segundo pueden consultarse los trabajos de J. Roche (1997: 4-11), L. Macé (2005: 93-104) y L. W. Marvin (2008: 244-258). 
embarcaciones se deslizaran a través de ellos y pudiesen salir del mar. En este caso concreto, para poner los troncos en el suelo hasta cerca de la muralla los combatientes catalanes y aragoneses se debieron proteger con algún mantelete o con cledes. A continuación, o a la vez que se depositaron en el suelo, se les untó con aceite o algún líquido que facilitase el deslizamiento. Jaime I continua explicando en el Llibre dels Feits (caps. 159-161) que para mover el castillo hasta el foso se clavaron dos áncoras en el suelo con la ayuda de unos mazos, y que a través de la rodela de las áncoras se introdujeron unas estacas muy fuertes que también se fijaron en el suelo. Entonces, a las estacas se les ataron unos motones con poleas en su interior para que la cuerda unida al castillo de madera pudiera correr sin problemas, de atrás hacía delante. De esa manera, al tirar de las cuerdas, el castillo se movería encima de los parales en la dirección inversa a la que se hacía la fuerza. Jaime I también explica que en la operación de clavar las áncoras en el suelo se puso delante un mantelete de cledes para proteger a los hombres que estaban trabajando. Asimismo, asegura que veinte combatientes con escudos gruesos protegieron a los hombres que tiraban de las dos cuerdas (Monreal y Tejada, 1971: 30-31).

La finalidad del castillo de madera era desalojar a los defensores del camino de ronda de la muralla. Para conseguir este fin el castillo debía ser más alto que el muro o torre a barrer para que los ballesteros del piso de arriba pudiesen disparar a placer sobre los sitiados. Una vez desalojada la muralla o torre de defensores, un grupo de asaltantes podía apoderarse de la posición enemiga.

\subsubsection{El asalto a la plaza asediada}

Cuando una torre o parte de la muralla se había derribado del todo o de manera parcial se procedía al asalto. En el de la ciudad de Mallorca (1229) la grieta hecha en la muralla era tan importante que junto a los hombres de a pie pudieron entrar también caballeros armados que decidieron la batalla, según narra el Llibre dels Feits (caps. 84-85). En este caso concreto los defensores habían hecho unas líneas muy apretadas e impenetrables gracias a sus lanzas, hasta el punto que los hombres de a pie no podían abrirse paso entre ellos. Entonces los caballeros intervinieron con la maniobra conocida como la volta, que seguramente consistía en hacer girar los equinos contra las líneas enemigas para conseguir con el trasero de los animales partir las lanzas de los adversarios o deshacer su formación. Sin embargo, en otras ocasiones los caballeros se veían obligados a desmontar para tratar de asaltar una ciudad, tal como se relata en la Canso de la Crozada (27: 184) cuando se recuerdan los inicios del asedio de Tolosa (1217). Los ballesteros cumplían con un papel fundamental en los asaltos de ciudades o castillos, en el Llibre dels Feits (cap. 268) se documenta que Jaume I conquistó la torre Baytala de la ciudad de Valencia (1238) haciendo servir a todos los que tenía en su ejército, acción que sería decisiva para el desenlace del asedio. Sin embargo, el asalto en toda regla era un elemento, si no extraño, al menos poco frecuente, puesto que cuando los defensores veían un trozo de muralla o torre en un estado cercano al hundimiento o conquistada por los sitiadores acostumbraban a capitular de la forma más digna posible. Hay que tener en cuenta que lamentablemente cuando los atacantes entraban por la fuerza en una ciudad o castillo no era frecuente respetar ni vidas ni propiedades de los defensores. A su vez, a los sitiadores tampoco les interesaba en exceso un asalto en toda regla, a no ser que tuviesen una superioridad muy clara, puesto que había un alto riesgo de perder muchos hombres en el intento. Por ejemplo, de los tres asedios estudiados a Jaime I, sólo en el de Mallorca (1229) se llegó a un asalto general con el consecuente saqueo indiscriminado. En cambio, en el de Borriana (1232) la población capituló cuando uno de los fundíbulos de Jaime I consiguió derribar una de las torres, mientras que en Valencia (1238) la población también pactó la rendición cuando Jaime I conquistó la torre Baytala. 
Al igual que para el caso de los trabuquetes, fundíbulos y otras piezas de artillería había gente especializada en su construcción, para gatas y castillos de madera sucedía otro tanto igual. En ocasiones, podía ser el mismo especialista que había hecho los trabuquetes. Al menos así aparece documentado en el Llibre dels Feits (cap. 157), donde se afirma que el maestro que construyó el castillo de madera en Borriana era también el que había hecho antes el trabuquete de Mallorca.

\section{LA DEFENSA DE LA CIUDAD}

En la mayoría de los casos, el peso de la defensa de una ciudad lo soportaban los hombres que habitaban en ella, auxiliados de manera activa, en no pocas ocasiones, por las mujeres que vivían con ellos. Sin ir más lejos, en el asedio de Tolosa (1218), la piedra lanzada por el trabuquete que acabó con la vida de Simón de Montfort fue disparada por un grupo de mujeres de la ciudad, según la Canso de la Crozada (35: 205). Incluso durante el asedio de Gallipoli (1306), explica Ramón Muntaner en su Crònica (cap. 227) que fueron las mujeres de los almogávares las que rechazaron a los genoveses que atacaban la villa, puesto que sólo había cien hombres disponibles. En otras ocasiones, tal como se puede apreciar en el Llibre dels Feits (cap. 84) cuando se realiza el asalto final a Mallorca (1229), Jaime I no distingue entre civiles y profesionales de la guerra, dejando claro que en el contexto de la defensa de una ciudad asediada había pocas diferencias entre unos y otros: «...toda la gente de los sarracenos de la ciudad...». A veces se dice explícitamente que la defensa de una ciudad estaba compartida por profesionales de la guerra y milicianos urbanos, como sucedió durante el gran asedio de Tolosa (1217-1218), donde si bien el peso principal de la defensa lo soportaron los de la villa, en todo momento éstos fueron ayudados por los caballeros del conde de Tolosa, de los Cumenge, de los Foix, del catalán Dalmau de Creixell, de Bernat de Casnac y del hijo del conde de Tolosa, Ramón VII el Joven. Parece que sólo en casos muy excepcionales la defensa de una ciudad la protagonizaban en exclusiva profesionales de la guerra. El único caso encontrado en la documentación analizada se produjo en la defensa de Girona (1285). Entonces, Pedro el Grande obligó a los gerundenses a abandonar la ciudad, para instalar en su lugar una guarnición compuesta de 80 caballeros, 20 ballesteros a caballo y 2.500 combatientes de a pie (servents) armados con lanzas y ballestas, entre los cuales había 600 ballesteros musulmanes del reino de Valencia. Todos ellos estaban bajo las órdenes de Ramón Folch de Cardona, según narra Bernat Desclot en su Crònica o Llibre del rei En Pere (cap. 153). El miedo a una rápida capitulación o traición, tal como había sucedido recientemente en Castelló d’Empúries, fue lo que impulsó al que era rey de Aragón, de Sicilia, de Valencia y conde de Barcelona a tomar una decisión tan drástica. Un factor que también influyó fue la imposibilidad de tener suficientes vituallas para alimentar a los habitantes de la villa y soldados profesionales durante un largo tiempo.

\subsection{ACONDICIONAMIENTO ÓPTIMO DE LAS DEFENSAS: AVITUALLAMIENTO Y REFUERZOS DE LA MURALLA}

Cuando se sabía que una ciudad iba a ser asediada lo primero que se hacía era asegurar el avituallamiento para aquéllos que se quedasen a defenderla, con la idea de que resistieran el máximo tiempo posible el ataque de los sitiadores. Así aparece explicado por Bernat Desclot en su Crònica o Llibre del rei En Pere (cap. 153) con motivo de los preparativos para el asedio de Girona (1285): «...reunió toda la vianda que pudo encontrar en la tierra, de Barcelona a más allá, y la introdujo en Girona, e introdujo carneros salados y cerdos vivos, y gallinas para los enfermos, y toda cosa que pudo encontrar que fuese para el establecimiento buena». El siguien- 
te paso era mejorar las fortificaciones. Para esto se delimitaba el espacio que se iba a defender y se destruían las partes consideradas imposibles de mantener para que el enemigo no las pudiese aprovechar. Bernat Desclot (cap. 153) relata cómo en este mismo asedio Ramón Folch abandonó los arrabales exteriores a la muralla, llevándose los tejados y vigas de madera de las casas abandonadas al interior del recinto amurallado. A continuación, las murallas de piedra y cal existentes se reforzaban con la construcción de barbacanas -obras de fortificación a modo de castillejos situadas delante de las puertas para aumentar su potencial defensivo- y buhardas -bastidas de madera que se ponían encima de las torres y muros para esconder a los defensores de la plaza. Además, existían otras fortificaciones provisionales que tenían la función de substituir una muralla, bien porque no había, o debido a que la existente era insuficiente para proteger toda la ciudad y los sitiados no querían desprenderse de aquellas partes que quedaban fuera del núcleo amurallado. En algunas ocasiones se hacían muros de cal y mortero, tal como se recoge en la Canso de la Crozada (20: 166), pero da la sensación que era más frecuente la construcción de barreras de madera o empalizadas. En el asedio de Tolosa (1217-1218), la empalizada se reforzó a su vez como si se tratase de un muro de piedra, con lisera-espacio de tierra que se dejaba entre la muralla y el foso para que las piedras desprendidas del muro no llenasen el foso- camino de ronda, foso, buharda, ventanales y arquerías para que pudiesen disparar los ballesteros, tal como se describe en la Canso de la Crozada (27: 183 - 28: 187). En otras ocasiones las barreras de madera se hacían como complemento defensivo de la muralla, tal como narra Bernat Desclot en su Crònica o Llibre del rei En Pere (cap. 156) para referirse a las medidas tomadas por Ramón Folch en la defensa de Girona, puesto que dice que delante del muro y de la barbacana se había hecho una pared seguramente de madera. Sin embargo, no siempre las murallas provisionales se hacían de este material, en esta misma obra se dice que para proteger Barcelona de un presumible ataque cruzado Pedro el Grande construyó un muro de tierra (cap. 157): «...el rey hizo hacer un foso alrededor de la ciudad, ancho y profundo, con muro de tierra que hizo hacer al lado del mar, todo agujereado de ballesterías. Y, de veinte en veinte brazas, hizo hacer buhardas de madera al lado del foso, y entre dos castillos hacía hacer una brigola». Como se puede comprobar en este último fragmento, para defender bien una ciudad también se requería piezas de artillería para contrarrestar las que tenían los sitiadores. Jaime I dice en su Llibre dels Feits (cap. 69) que durante el asedio de la ciudad de Mallorca los sitiados disponían de dos trabuquetes y catorce algarradas, y que una de estas últimas era tan potente que incluso las piedras que lanzaba llegaban al campamento cristiano.

\subsection{FunCIONES DE LA GUARNICIÓN ASEDIADA}

\subsubsection{Hostigamiento de los sitiadores}

Uno de los cometidos que debía cumplir la guarnición asediada era realizar salidas al exterior de la plaza para dificultar a los sitiadores las obras de asedio e interceptar su avituallamiento siempre que fuese posible. Por ejemplo, Jaime I explica en el Llibre dels Feits (cap. 156) que los musulmanes de Borriana hacían incursiones fuera de la ciudad para capturar las vituallas que llegaban por vía marítima al campamento catalán y aragonés. Así se lograban dos objetivos a la vez, un extra de comida para la guarnición y debilitar al ejército atacante al privarle de la alimentación necesaria. Afirma Ramón Muntaner en su Crònica (cap. 128) que durante el asedio de Girona (1285) la guarnición catalana impidió el sueño y la comida al ejército francés gracias a sus ataques sorpresa al campamento cruzado. En algunos casos se instalaban contingentes de caballeros y hombres a pie en castillos y puntos fuertes cercanos 
a la ciudad asediada con la misión de cortar el avituallamiento a los sitiadores y hostigarlos continuamente. En este mismo asedio de Girona, Bernat Desclot relata en su Crònica o Llibre del rei En Pere (cap. 157) que Pedro el Grande instaló caballeros catalanes y servents en las villas de Besalú y Hostalric para que llegasen con sus correrías hasta el campamento cruzado e impidiesen la llegada de vituallas.

\subsubsection{La defensa del recinto amurallado}

Sin lugar a dudas, el principal cometido de la guarnición asediada era el de rechazar los ataques del ejército sitiador. A través de la información aparecida en las fuentes analizadas se puede concluir que los defensores se dividían en grupos que ocupaban los diferentes tramos de la muralla, acompañados de botas de vino y cestas con pan para que los soldados que tuviesen hambre y sed no abandonasen sus puestos. En el camino de ronda también se distribuían piedras y ollas con agua hirviendo o líquidos inflamables para ser vertidos sobre los asaltantes. Tal como relata Ramón Muntaner en su Crònica (cap. 227), unos cuantos defensores se quedaban como reserva móvil para socorrer aquellos tramos más amenazados por el enemigo o hacer salidas afuera que contrarrestasen su ataque. Si la guarnición que protegía una ciudad era numerosa se podía permitir el lujo de dividir sus fuerzas en dos, para que mientras los ballesteros desde las buhardas disparasen contra los asaltantes a través de las ballesterías y ventanales, el resto de combatientes saliesen fuera de la muralla y ocupasen las liseras para evitar así que los atacantes se acercasen al pie del muro, tal como aparece descrito en la Canso de la Crozada (28: 187).

\subsubsection{Acciones para contrarrestar el efecto de los picadores}

Para contrarrestar a aquéllos que picaban el pie de la muralla existían fórmulas originales. Según la Canso de la Crozada (19: 164), durante el asedio de Beaucaire del año 1216, los defensores introdujeron azufre y estopa dentro de un trapo cosido que fue descendido muralla abajo con la ayuda de una cadena. Antes del descenso se había encendido la estopa, de manera que cuando el trapo llegó al foso el fuego ya había consumido la estopa y había entrado en contacto con el azufre, provocando una gran llamarada que obligó a los picadores huir. En varias ocasiones, como se ha visto con anterioridad, para picar una muralla era necesario llenar el foso con pertrechos. Para destruirlos se podía hacer de la manera más sencilla, lanzando desde la muralla una tea o antorcha encendida, aunque existían otros métodos más elaborados, como construir una mina debajo de ellos para posteriormente encenderles fuego, tal como relata Jaime I que hicieron los musulmanes de Mallorca (1229) en su Llibre dels Feits (cap. 73).

\subsubsection{Acciones para contrarrestar las minas}

Para contrarrestar la acción de las minas se construían otras dentro de la ciudad que tenían la finalidad de interceptar las que habían hecho los sitiadores. En ocasiones, cuando la contramina hecha por los sitiados chocaba con la de los atacantes se producían auténticas batallas subterráneas, tal como se recoge en el Llibre dels Feits (cap. 73) para el asedio de la ciudad de Mallorca (1229). Otra forma de hacer inútil el esfuerzo de hacer una mina era mediante la construcción en secreto de un muro detrás de la muralla que debía ser derribada, como el que hizo Ramón Folch durante el asedio de Girona (1285) para frustrar el intento de los cruzados y que recoge Bernat Desclot en su Crònica o Llibre del rei En Pere (cap.161). 


\subsubsection{Acciones para contrarrestar las máquinas de aproximación}

Para hacer frente a las máquinas o instrumentos que se acercaban a la muralla se hacían servir diferentes procedimientos. En general, el fracaso o éxito de este tipo de ataques dependía de la superioridad en ballesteros que tuviese uno de los dos contendientes. Tanto el castillo de madera construido por Jaime I en Borriana (1232), como los que hicieron los cruzados franceses en Girona (1285), fracasaron por los dardos que tiraron los ballesteros defensores (Monreal y Tejada, 1971: 30-32). En el primero de los casos, los musulmanes dispararon con tanta intensidad contra los hombres que manipulaban las cuerdas conectadas al castillo de madera que, a pesar de estar protegidos con grandes escudos, se vieron obligados a desistir de su intento. A continuación, el castillo inmovilizado fue destruido con las piedras lanzadas por las algarradas de los defensores, tal como se recoge en el Llibre dels Feits (cap. 161-163). En el segundo debió pasar algo parecido, aunque Bernat Desclot únicamente narra en su Crònica o Llibre del rei En Pere (cap. 163) que el ataque de los cruzados fracasó debido a la acción de los ballesteros sarracenos de la guarnición catalana, puesto que imposibilitaron a los franceses asomar las manos o las cabezas fuera de los castillos de madera. A las gatas e instrumentos similares había varias maneras de neutralizarlos. Parece que una de las más utilizadas era la técnica de tirarles agua hirviendo o líquidos inflamables desde la parte alta de la muralla a través de una olla, tal como se relata en la Canso de la Crozada (13: 134). Más adelante, en esta misma obra (21: 169) aparece otra variante de esta misma tipología que consistía en lanzar agua mezclada con cal hirviendo. En la Canso de la Crozada (20: 167) se documenta también una tercera forma para abortar los ataques producidos con gatas e instrumentos similares, como era llenar una olla de alquitrán, incendiarla y abocar el líquido inflamable muralla abajo contra las máquinas de los sitiadores. En concreto, durante el asedio de Beaucaire (1216) los occitanos hicieron servir este procedimiento contra una mostela, que vendría a ser una gata pequeña: «Mientras, observad la mostela que comienza a sacar un pico; / el buen ingeniero, con buen corazón y generoso, / prendió fuego al alquitrán y la olla la llenó, / e hirió a la mostela directamente allí donde la vio ${ }^{13}$; / que la antorcha se incendió y el fuego se esparció / por todos lados, que con dificultades se apagó». Probablemente haya que interpretar la antorcha aparecida en el fragmento como los pertrechos que se lanzaban previamente al foso para poder picar los muros, que al entrar en contacto con el alquitrán vertido desde la muralla se habrían quemado provocando el abortamiento del ataque. Vuelve a ser de nuevo la Canso de la Crozada (34: 203 - 35: 204) quien informa de otra técnica para acabar con una máquina enemiga, concretamente mediante los golpes de piedra lanzados por los trabuquetes, procedimiento que utilizaron los defensores de Tolosa para neutralizar la gran gata construida por Simón de Montfort. Sin embargo, se podía lograr el mismo resultado de forma más simple, tal como hizo la guarnición de Girona para destruir las gatas francesas durante el asedio del año 1285, suceso narrado por Bernat Desclot en su Crònica o Llibre del rei En Pere (cap. 162). El cronista catalán relata que Ramón Folch hizo salir a 500 de sus hombres con teas en una mano y botijos llenos de aceite en la otra aprovechando la oscuridad de la noche. Llegados al lugar donde estaban las gatas, las untaron con el aceite que llevaban para incendiarlas a continuación (Monreal y Tejada, 1971: 28). Es de suponer que para contrarrestar la acción de los arietes se harían servir los mismos procedimientos que para las gatas, aunque en la Canso de la Crozada (19: 164) se ha dejado constancia de otras técnicas, como fijar la cabeza de la viga que golpeaba el muro con la ayuda de un lazo, acción que llevaron a cabo los defensores de Beaucaire en el año 1216. vig”.

13 Corrijo la traducción de V. Martines y G. Ensenyat. El texto original dice así: "E firit la mostela tot dreit la on la 


\subsubsection{Acciones para contrarrestar un asalto con escaleras}

Para abortar un asalto con escaleras se hacía servir el lanzamiento de piedras, de la manera más sencilla, cogiéndolas con las manos y dejándolas caer, o el tiro con las ballestas. Sin embargo, existían unos instrumentos que recibían el nombre de llebreres en catalán y que estaban diseñados precisamente para abortar ataques de estas características. Por lo que se puede interpretar de la descripción que hizo Bernat Desclot en su Crònica o Llibre del rei En Pere (cap. 164), sólo se necesitaba una gran viga vaciada por dentro y dos grandes muelas de molino para construirla. Cada extremo de la viga se introducía en el orificio central que tenía la muela de molino, de manera que al cargar la viga hueca con piedras y hacer rodar las muelas, todo el contenido caía encima de los que pretendiesen subir a la muralla ${ }^{14}$. De creer a Bernat Desclot, gracias a estas máquinas pudo Ramón Folch abortar el intento de asalto francés en Girona (1285).

\section{CONCLUSIONES}

Hasta aquí la información proporcionada por las fuentes estudiadas. El paso del tiempo es cruel con los materiales perecederos como las maderas y las fibras vegetales. En consecuencia, no ha llegado a la actualidad ningún testimonio material de un trabuquete, un fundíbulo, una algarrada, un castillo de madera o una llebrera. La interpretación de las breves descripciones de los cronistas es el único dato con el que cuenta el historiador para proceder a su reconstrucción, con el peligro que eso conlleva. Ciertamente, los autores de estas obras recogen importantes y ricas informaciones que se han analizado en este artículo, pero omiten otras no menos fundamentales que las anteriores, quizá porque el público contemporáneo a ellos no las necesitaba por conocerlas de sobra, que no es el caso del estudioso del siglo XXI lamentablemente. Por poner un ejemplo, las obras analizadas muestran claramente el poder destructor de un trabuquete de contrapeso, pero no señalan con claridad cómo se volvía a traer la vara después de realizar un primer disparo, acción que era realmente complicada, puesto que se debía levantar el contrapeso. Seguramente, lo hacían con un torno de eje vertical, como señala Villard de Honnecourt (Lám. 59: 137). No obstante, el cómo conseguían mantener aquellas varias toneladas de peso suspendidas en el aire sin que las cuerdas del cabrestante cediesen o el cómo se dejaban ir para efectuar el disparo no queda explicitado (Chevedden, 2000: 115-116). En este sentido, los trabajos de reconstrucción se han centrado más en dar una explicación física a la trayectoria del proyectil arrojado que al proceso de carga y descarga (Chevedden et alii, 1995: 66-71). La arqueología sí que puede contribuir de forma decisiva en el estudio de las estructuras defensivas, tales como fosos, murallas, torres o castillos. Contrastar los datos proporcionados por las fuentes escritas con los que son fruto de la excavación o estudio arqueológico debería ser un imperativo para la investigación militar del Medievo, aunque no puede ser sencillo distinguir del paramento de una determinada muralla qué es lo que se añadió a finales del siglo XIII con lo del primer cuarto del XIV.

Las obras analizadas muestran que el siglo XIII es en esencia una etapa de transición. La relativa facilidad con la que los sitiadores pudieron picar las murallas de Béziers (1209), Le Pujol (1213) o Valencia (1238), indica la poca presencia de matacanes o taludes inclinados en algunas fortificaciones de aquella época, a pesar que es hacia el año 1200 cuando se generalizan tales estructuras (Jones, 2010: 225). Asimismo, técnicas que pueden ser consideradas primarias, a pesar de su gran eficacia, como el lanzamiento manual de piedras o el picado de los

14 L. Monreal y Tejada (1971: 21) ofrece una descripción distinta de este instrumento: “consisten en una gran viga en cuyos extremos se ponen sendas ruedas de molino y se cargan con todas las piedras que se pueda”. 
muros, convivían con otras más sofisticadas, como el uso controlado y premeditado del fuego, el minado o los trabuquetes de contrapeso y demás piezas de artillería. Otra característica no menos importante es la aplicación de técnicas de la industria civil en fenómenos bélicos. La forma como se puso en movimiento el castillo de madera construido por Jaime I durante el asedio de Borriana (1232), no era ni más ni menos que un calco del método que hacían servir los marineros para sacar las embarcaciones del mar, tal como se puede apreciar en el Llibre dels Feits (cap. 161).

Sin embargo, más allá de una simple numeración y descripción de las tecnologías utilizadas en los asedios del siglo XIII, el análisis de las fuentes escritas puede ofrecer datos de mayor trascendencia historiográfica. La gran importancia de la infantería, muy especialmente de los ballesteros, tanto en las fases ofensivas como defensivas de los sitios, en contraste con el papel simplemente auxiliar o de apoyo de la caballería en los mismos, debe hacer replantear la hegemonía militar de esta última en el campo de batalla medieval, más todavía si tal como afirman J. Bradbury (1992: 71) o R. L. C. Jones (2010: 212) la mayor parte de acciones bélicas de esta época transcurrían en asedios de ciudades o castillos. De la quincena de sitios estudiados para la realización de este artículo, sólo en la conquista de la ciudad de Mallorca (1229) los caballeros pudieron participar en el asalto definitivo montados en los lomos de sus animales, tal como se recoge en el Llibre dels Feits (caps. 84-85). En los demás, por el contrario, o tuvieron un papel secundario en el resultado final del enfrentamiento, o se vieron obligados a desmontar de sus caballos, perdiendo entonces buena parte de su potencia bélica. De la misma manera, la existencia de un personal técnico altamente cualificado para el diseño y construcción de máquinas de guerra invalida uno de los tópicos más extendidos en el subconsciente popular, el de la presunta decadencia tecnológica del Medievo en comparación con otras etapas históricas. Ciertamente, los guerreros que participaron en estos asedios en más de una ocasión tuvieron una actuación bárbara, con todo el sentido peyorativo del término, pero no por eso dejaba de ser una barbarie inteligente, muy a pesar de sus víctimas.

\section{BIBLIOGRAFÍA}

FUENTES

CANSO DE LA CROZADA: Martin-Chabot, E. (Ed. y Trad. Fr.) (1931-1961): La Chanson de la Croisade Albigeoise. 3 vols. París, Libr. Honoré Champion, Les Belles Lettres. Martines, V., Ensenyat, G. (Trad. Cat.) (2003): Cançó de la croada contra els albigesos. Barcelona, Proa.

CRònICA O LLIBRE DEL REI EN PERE DE BERNAT DeCLOT: Cingolani S. M. (Ed.) (2010): Llibre del rei En Pere. Barcelona, Editorial Barcino.

CRòniCa de RAmon Muntaner: Soldevila, F., Bruguera, J., Ferrer i Mallol, M. T. (Ed. y Rev.) (2011): Les quatre grans Cròniques. III. Crònica de Ramon Muntaner. Barcelona, Institut d'Estudis Catalans.

CUADERNo de VILLARD DE HonNeCourt: Erlande-Brandenburg, A., Pernoud, R., Gimpel, J., Bechmann, R. (Ed. y Trad. Es.) (1991): Cuaderno. Siglo XIII. A partir del manuscrito conservado en la Biblioteca Nacional de París (n.19093). Madrid, Akal.

LLIBRE DELS FEITS DEL REI EN JAUME: Soldevila, F., Bruguera, J., Ferrer i Mallol, M. T. (Ed. y Rev.) (2007): Les quatre grans Cròniques. I. Llibre dels feits del rei En Jaume. Barcelona, Institut d'Estudis Catalans.

MONOGRAFÍAS

Alcover, A. M. y Moll, F. de B. (2001-2002): Diccionari català-valencià-balear. Ed. Electrónica. Institut d'Estudis Catalans, Editorial Moll. 
Alvira Cabrer, M. (2002): 12 de Septiembre de 1213. El Jueves de Muret. Barcelona, Universitat de Barcelona.

Alvira Cabrer, M. (2010): “Aspects militaires de la Croisade albigeoise”. Au temps de la Croisade. Société et pouvoirs en Languedoc au XIIIe siècle. Carcassone, Conseil général de l'Aude: 59-72.

Berlioz, J. (1994): Tuez-les tous. Dieu reconnaîtra les siens. La Croisade contre les Albigeois vue par Césaire de Heisterback. Portet-sur-Garonne, Loubatières.

Bourin, M. (Dir.) (2010): En Languedoc au XIIIe siècle. Le temps du sac de Béziers. Perpignan, Presses Universitaires de Perpignan.

Bradbury, J. (1992): The Medieval Siege. Woodbridge, Boydell \& Brewer.

Bruhn de Hoffemeyer, A. (1982): Arms \& Armours in Spain. II. A short survey. Madrid, Instituto de Estudios sobre Armas Antiguas.

Chevedden, P. E., Eigenbrod, L., Foley, V., Soedel, W. (1995): “The Trebuchet: Recent Reconstructions and Computer Simulations reveal the Operating Principles of the Most Powerful Weapon of its Time”. Scientific American, CCLXXIII/1: 66-71.

Chevedden, P. E. (1996): "The Artillery of King James I the Conqueror". Iberia and the Mediterranean World of the Middle Ages. II (ed. P. E. Chevedden, D. Kagay, P. Padilla). Leiden, Brill: 47-94.

Chevedden, P. E. (2000): "The Invention of the Counterweight Trebuchet: A Study in Cultural Diffusion”. Dumbarton Oaks Papers, LIV: 71-116.

Chevedden, P. E. (2011): "King James I the Conqueror and the artillery revolution of the Middle Ages". Jaume I. Commemoració del VIII centenari del naixement de Jaume I. I (ed. M. T. Ferrer i Mallol). Barcelona, IEC: 313-339.

Compan, A. (1989): “Étude des termes obsidionaux dans la Croisade des Albigeois”. Bulletin du Centre de romanistique et de latinité tardive, IV/5: 45-61.

Contamine, Ph. (1984): La guerra en la Edad Media. Barcelona, Editorial Labor.

Corfis, A. y Wolfe M. (1995): The Medieval City under Siege. Woodbridge, The Boydell Press.

Finó, J. F. (1972): “Machines de Jet Médiévales”. Gladius, X: 25-43.

García Fitz, F. (1997): “Tecnología militar y guerra de asedios. La experiencia castellano-leonesa, siglos XI al XIII”. Military Studies in Medieval Europe (ed. Guy De Boe y Frans Verhaeghe). Zellik, Instituut voor het Archeologisch Patrimonium: 38-41.

García Fitz, F. (2000): "El cerco de Sevilla: reflexiones sobre la guerra de asedio en la Edad Media". Sevilla 1248. Congreso Internacional conmemorativo del 750 aniversario de la conquista de Sevilla por Fernando III, Rey de Castilla y León. Sevilla, Centro de Estudios Ramón Areces: 115-154.

García Fitz, F. (2001): Castilla y León frente al Islam. Estrategias de expansión y tácticas militares (siglos XI-XIII). Sevilla, Universidad de Sevilla.

García Fitz, F. (2005): “¿Machinis validas? Tipología y funcionalidad de las máquinas de asedio en el medievo hispano. Castilla-León, siglos XI al XIII”. Actas del III Congreso de Castellología Ibérica (coord. Amador Ruibal). Madrid, Asociación Española de Amigos de los Castillos, Diputación Provincial de Palencia: 219-254.

García Fitz, F. (2011): “Tecnología, literatura técnica y diseño de máquinas de guerra durante la Baja Edad Medía occidental: el Thexaurus regis Franciae acquisitionibus Terrae Santae de Guido da Vigevano (1335)". Anuario de Estudios Medievales, XLI/2: 819-864.

Gillmor, C. M. (1981): "The Introduction of the Traction Trebuchet into the Latin West". Viator, XII: $1-8$.

Hélas, J. C. (2001): “Attaque et défense des châteaux et des villes fortifiées à partir des trois principales sources de la croisade des Albigeois”. Acta Archaelogica Lodziensia, XLVII: 51-60.

Higham, R., Barker, P. (1992): Timber Castles. Londres, Batsford.

Hill, D. R. (1973): "Trebuchets". Viator, IV: 99-115.

Hill, D., Rumble, A. (1996): The Defense of Wessex: The Burghal Hidage and Anglo-Saxon Fortifications. Manchester, Manchester University Press.

Humphries, P. D. (1985): "Of Arms and Men: Siege and Battle Tactics in the Catalan Grand Chronicles (1208-1387) ". Military Affairs, XLIX/4: 173-178.

Jones, R. L. C. (2010): “Fortalezas y asedios en Europa occidental c. 800-1450". Historia de la guerra en la Edad Medía. Madrid, Machado Libros: 211-238.

King, D. J. C. (1982): “The Trebuchet and other Siege-Engines”. Château Gaillard, IX/10: 457-469. 
Ladero Quesada, M. A. (coord.) (2010): Historia Militar de España, T.II: Edad Media. Madrid, Laberinto.

Langlois, G. (1994): "Le siège du château de Termes par Simon de Montfort en 1210. Problèmes topographiques et historiques”. Heresis, XXII: 101-134.

Langlois, G. (1995): "Le siège du château de Termes. Addition et correction". Heresis, XXIV: 87-89.

Langlois, G. (2005): "La guerre des châteaux. Le siège de Termes (1er août-23 novembre 1210". Les grandes batailles méridionales, 1209-1271. Toulouse, Privat: 53-60.

Macé, L. (2005): "La croix fait des progres et le lion perd du terrain. Le siège de Beaucaire (fin mai-24 août 1216) ". Les grandes batailles méridionales, 1209-1271. Toulouse, Privat: 93-104.

Martin, R. (2012): Dictionnaire du Moyen Françaies (1330-1500). Ed. Electrónica. Nancy, ATILF.

Marvin, L. W. (2001): "War in the South: A First Look at Siege Warfare in the Albigensian Crusade, 1209-1218”. War in History, VIII: 373-395.

Marvin, L. W. (2008): The Occitan War. A Military and Political History of the Albigensian Crusade, 1209-1218. Cambridge, Cambridge University Press.

Meschini, M. (2005): "Pourquoi Béziers? La chute de Béziers (22 juillet 1209)". Les grandes batailles méridionales, 1209-1271. Toulouse, Privat: 25-38.

Monreal y Tejada, L. (1971): Ingeniería militar en las crónicas catalanas. Barcelona, Real Academia de Buenas Letras de Barcelona.

Morillo, S. (1994): Warfare under the Anglo-Norman Kings, 1066-1135. Woodbridge, Boydell Press.

Navareño Mateos, A. (1998): "El castillo en la guerra medieval. Pertrechos y tácticas de ataque y defensa”. Actas del I Congreso de Castellología Ibérica. Palencia, Diputación Provincial de Palencia: $575-592$.

Peytavie, C. (2005): "Le siège de Toulouse (octubre 1217-juillet 1218)". Les grandes batailles méridionales, 1209-1271. Toulouse, Privat: 105-124.

Prestwich, M. (1996): Armies and Warfare in the Middle Ages: The English Experience. New Haven y Londres, Yale University Press.

Purton, P. F. (2009): A History of the Early Medieval Siege, c. 450-1220. Woodbridge, Boydell \& Brewer.

Purton, P. F. (2010): A History of the Late Medieval Siege, c. 1220-1500. Woodbridge, Boydell \& Brewer.

Roche, J. (1997): “Autour du siège de 1216: les ambigüités du récit”. Bulletin de la Société d'histoire et d'archéologie de Beaucaire, CXXXV: 4-11.

Rogers, R. (1992): Latin Siege Warfare in the Twelfth Century. Oxford, Clarendon Press.

Roquebert, M. (2001): L'Épopée Cathare. I. Toulouse, Perrin-Privat.

Runciman, S. (1965): The Fall of Constantinople 1453. Cambridge, Cambridge University Press.

Vazeille, J. B. (1962-1963): "La prise de Pujol: signification de cet épisode à la veille de Muret". Annales de l'Institut d'Études Occitanes: 124-132.

Zambon, F. (2006): "Descrizioni di assedi nella Canzone della crociata albigese di Guilhem de Tudela". Medioevo romanzo, XXX/1: 24-37.

Recibido: 10/11/11

Aceptado: 21/06/13 